\title{
Response of borehole SOTEM on two-dimensional earth
}

\author{
Weiying Chen, Guoqiang Xue \\ Key Laboratory of Mineral Resources, Institute of Geology and Geophysics \\ Chinese Academy of Science \\ Beijing, China
}

\begin{abstract}
In this paper, we proposed a novel TEM device-borehole SOTEM based on the advantages of borehole TEM and ground SOTEM. In order to get a preliminary understanding to this method, we calculated the response on two-dimensional earth using FDTD scheme and analyzed its characteristics as well. The results showed that distinct and easily identifiable change law contained both in static and dynamic characteristics of response curves, which can be used to identify the location and electrical property of ore bodies.
\end{abstract}

Keywords-borehole; SOTEM; 2D; response

\section{INTRODUCTION}

The working space for transient electromagnetic methods (TEM) can be airborne, ground and underground. Generally, the underground TEM has the higher detection precision and greater detection depth due to the smaller distance between receivers and targets ${ }^{[1]}$. At present, the borehole TEM devices are mostly based on ground loop transmitter (magnetic source) $[2-5]$

Using a grounded wire (electric source) as transmitter is another working form for TEM. At present, the working space for electric source TEM not only includes the ground ${ }^{[6-7]}$, but also the airborne ${ }^{[8]}$ and the marine ${ }^{[9]}$. However, the research and application of in-borehole has not discussed. In view of the advantages of ground SOTEM and borehole TEM method, it is very necessary to study the feasibility of borehole SOTEM. In this paper we proposed a method which uses a grounded wire source and observes signal in drill hole. Then we analyzed the characteristics of the response of $2 \mathrm{D}$ earth models. The purpose of our study is to give a brief understanding of this method and build a foundation for the further studies.

\section{INTRODUCTION OF BOREHOLE SOTEM}

In borehole SOTEM, a grounded wire is laid on the earth surface and input bipolar step current, and the signal is recorded by special probes from shallow to deeper levels along the well. In order to reduce the volume effect and enhance signal noise ratio, transmitter is generally apart from the wells in a distance with tens to hundreds meters. At the same time, in order to get the optimum coupling between transmitter and ore bodies, we can lay multiple transmitters at different locations around the well and observe the signal from each source successively. The diagrammatic sketch of surface to borehole SOTEM is shown in Fig. 1.

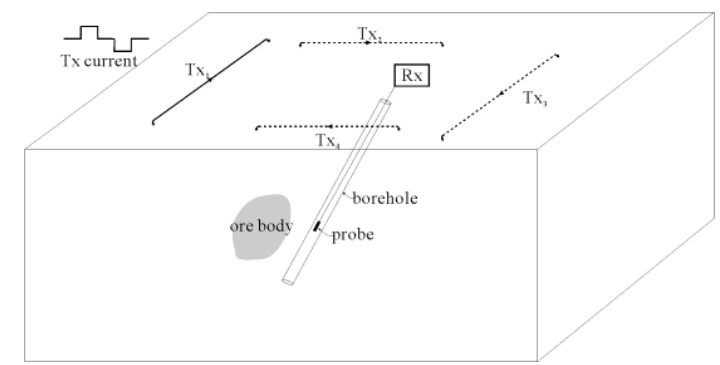

Fig.1. Diagrammatic sketch of surface to borehole SOTEM

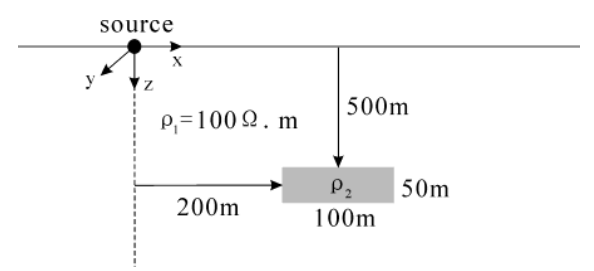

Fig.2. Sketch of 2D model

\section{FDTD METHOD}

Finite difference in time domain (FDTD) method is an effective tool for solving the TEM forwarding problems of 2D media ${ }^{[10-11]}$. To calculate transient field the first step is dispersing the partial differential equation at a certain time with difference methods to calculate the values at the current time, and then deduce the values of next moment.

In our study, a 2D model shown in Fig. 2 was designed. An infinite plate along the $\mathrm{Y}$ direction is inputed in a homogeneous halfspace with resistivity of $100 \Omega$.m. The resistivity of the plate is $10 \Omega . \mathrm{m}$ for conductive abnormal body and $1000 \Omega$.m for resistive abnormal body. The position and geometry size of the abnormal body as well as the position of transmitter as shown in Fig. 2.

\section{ANALYSIS OF RESPONSE CHARACTERISTICS}

The characteristics of downhole TEM response can be classified as static characteristics and dynamic characteristics. The so-called static characteristics also known as spatial characteristics or profile curve characteristics, mainly reflects the influence from the coupling relationship between the source and targets to anomaly shape and distribution. The so-called dynamic characteristics also known as time characteristics, mainly reflects the distribution and decay of induced eddy current in the anomaly body with the time change. The study of these two characteristics contributes to the qualitative analysis of feasibility of borehole SOTEM and 
provide theoretical basis for the subsequent data processing and interpretation.

\section{A. Static characteristics}

The relative position of transmitter, targets and wells usually cannot be determined beforehand in the field work. In order to analyze the influence of relative position, we calculated the response profiles at different offsets as shown in Fig. 3. The offset (1) and (2) represent the well is located between the source and plate; the offset (3), (4) and (5) represent the well pass through the plate; the offset (6) and (7) represent the well is located at the outside of the plate. The time of profiles is $100 \mathrm{~ms}$.

Firstly, it can be seen from Fig.3 that the vertical induced voltage of borehole SOTEM shows more obvious reflection to conductive abnormal body (Fig. 3a) than resistive abnormal body (Fig. 3b). Moreover, the profile curves of conductive model diverge to both sides of the abnormal body while the curves converge to the center of abnormal body for resistive model. The response profiles with different offsets also show variations in different degrees and different directions. For the conductive abnormal body, with the measuring point closing the abnormal body, the amplitude of response decrease (possibly negative anomalies) when the well is located between the source and the abnormal body or pass through the near-source part of abnormal body, while the amplitude increase when the well is located outside the abnormal body or pass through the away-part of abnormal body. For resistive abnormal body, the response profiles just show opposite variation characteristics. It also can be seen from Fig. 3 that the profile curves don't show the biggest anomaly when the well pass through the center of the abnormal body, which can be explained as the distribution of eddy current is more uniform than its edge zones.

The above analysis indicate that the location, depth and electrical prosperity of ore bodies can be obtained according to the response profiles from different coupling relations between transmitter, well and ore bodies.

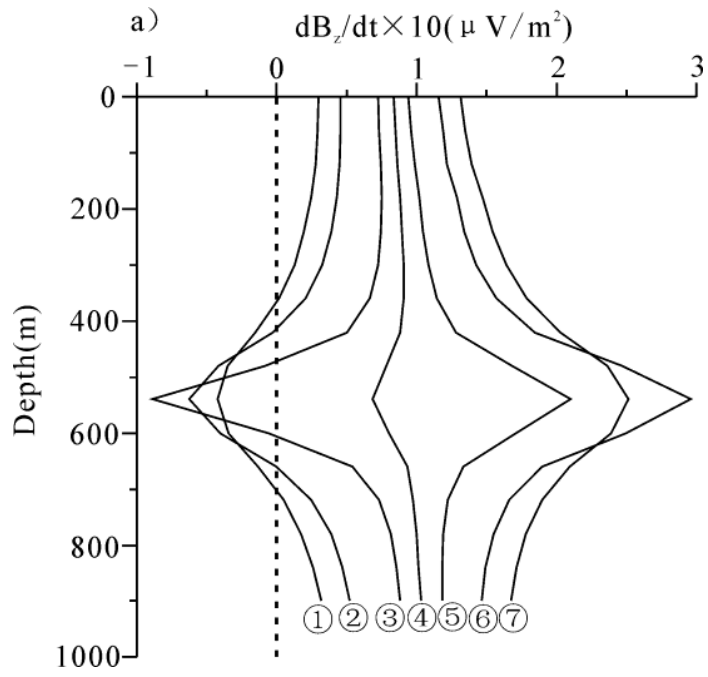

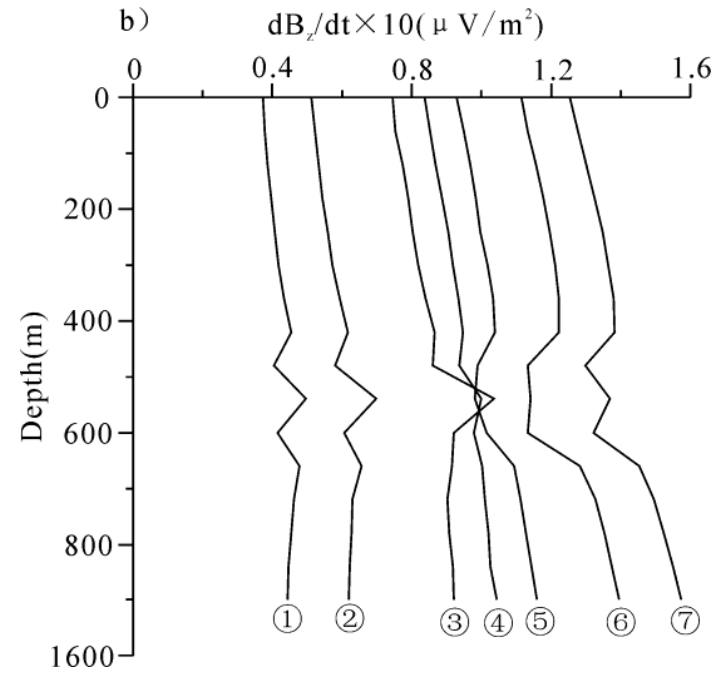

Fig.3. Response profiles of different offset at 100ms. (a)Conductive abnormal body,(b) resistive abnormal body. The members at the ends of curves represent different offsets: (1) $\mathrm{r}=102.4 \mathrm{~m}$, (2) $\mathrm{r}=149.7 \mathrm{~m}$, (3) $\mathrm{r}=228.5 \mathrm{~m}$, (4) $\mathrm{r}=260 \mathrm{~m}$, (5) $\mathrm{r}=291.5 \mathrm{~m}$, (6) $\mathrm{r}=354.5 \mathrm{~m}$, (7) $\mathrm{r}=401.8 \mathrm{~m}$

\section{B. Dynamic characteristics}

In the practical measurement, borehole SOTEM measures the signal within a period of time at different depth along the drill. Like ground TEM, the response of borehole SOTEM can also be divided into early, middle and late times. Fig. 4 indicates response curves measured at different depth for conductive model. Three offsets are considered, among which the Fig. 4a represents the well is located between the transmitter and the abnormal body, Fig. $4 \mathrm{~b}$ represent the well roughly pass through the center of the abnormal body and Fig. $4 c$ represents the well located away from the abnormal body. Some analogous characteristics can be obtained from the results at three offset. First, the early time responses increase with time when measuring point at greater depth. Second, the middle and late times curves show different changes when the observation point and abnormal body are placed at the same depth $(d=520 m)$. However, these changes are different for different offsets. Specifically speaking, the response turns to be negative when the well is located between the source and the abnormal body, the response decays faster when the well passes through the anomaly body but there is no sign changing, and the signal strength becomes higher than other depths when the well is located outside the anomaly body. Thus, according to the dynamic characteristics of borehole SOTEM response, especially when receiving point and abnormal body in the same depth, we can obtain the rough information of position and electric property of targets. 

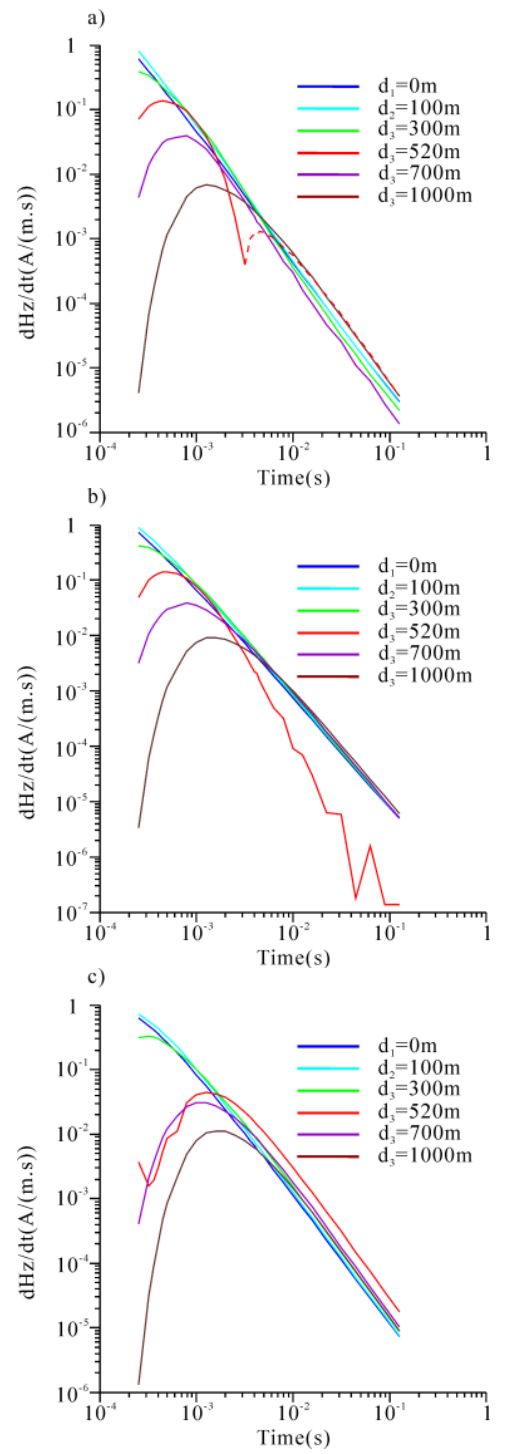

Fig.4. Decay curves of borehole SOTEM at different depths. (a) r=149.7m, (b) $r=244.2 \mathrm{~m},(\mathrm{c}) \mathrm{r}=354.5 \mathrm{~m}$

\section{CONCLUSIONS}

In view of the superiority of borehole TEM and ground SOTEM, this paper presented a new type of downhole TEM device - borehole SOTEM, and then studied its response on a two-dimensional earth by using FDTD method. Some useful conclusions were obtained as follows:
1. The vertical magnetic field component is more sensitive to the conductive targets than resistive targets, which is the same as the ground TEM method.

2. The borehole profiles show different variations if different geometric coupling relationships are considered among the source, abnormal body and well. These differences of static characteristics qualitatively tell us the position and electrical property of the abnormal bodies.

3. The borehole SOTEM responses observed at different locations also show different dynamic characteristics, especially when the depth of the receiving point is consistent with the ore body, the response curves show a significant difference compared with the other depths.

\section{REFERENCES}

[1] Dyck A V. 1991. Drill-hole Electromagnetic Methods, in Nabighian M M, ed., Electromagnetic Methods in Applied Geophysics, SEG: 881-929.

[2] Eaton P A. and Hohmann G W. 1984. The influence of a conductive hoston two-dimensiona borehole transient electromagnetic response. Geophysics, 49(7): 861-869.

[3] West R C. and Ward S H. 1988. The borehole transient electromagnetic response of a three-dimensional fracture zone in a conductive half-space, Geophysics, 53(11): 1469-1478.

[4] Meng Q X, Pan H P. 2012. Numerical simulation analysis of surface-hole TEM responses. Chinese J. Geophy. (in Chinese), 55(3): 1046-1053.

[5] Zhang J, Deng X H, Tan H D, et al. 2015. A study of vector intersection for borehole transient electromagnetic method. Geophysical and Geochemical Exploration, 39(3): 572-579.

[6] Strack, K M. 1992. Exploration with deep transient electromagnetic method: Elsevier.

[7] Chen W Y, Xue G Q, Muhammad Y K, et al. 2015. Application of short-offset TEM (SOTEM) technique in mapping water-enriched zones of coal stratum, an example from East China. Pure and Applied Geophysics, 172(6): 1643-1651

[8] Mogi T, Kusunoki K, Kaieda H, et al. 2009. Grounded electrical-source airborne transient electromagnetic (GREATEM) survey of Mount Bandai, north-eastern Japan. Exploration Geophysics, 40: 1-7.

[9] Constable S. and Weiss C. J.. 2006. Mapping thin resistors and hydrocarbons with marine EM methods: insight from 1D modeling. Geophysics, 71(2): G43-G51.

[10] Oristaglio M L, Hohmann G W. 1984. Diffusion of electromagnetic fields into a two dimensional earth: A finite-difference approach. Geophysics, 49(7): 870-894.

[11] Yan S, Chen M S, Fu J M. 2002. Direct time-domain numerical analysis of transient electromagnetic fields. Chinese J. Geophy. (in Chinese), $45(2): 275-284$. 